\title{
El progreso de la ciencia como resolución de problemas: una defensa de las posturas funcionalistas-internalistas
}

\author{
Damián Islas Mondragón \\ Universidad Juárez del Estado de Durango
}

Resumen

Recientemente, Alexander Bird (2007) sugirió que la ciencia progresa cuando muestra "acumulación de conocimiento justificado". Para validar su postura, Bird contrastó sus ideas con los conceptos sobre el progreso científico construidos por Thomas S. Kuhn y Larry Laudan, respectivamente. El objetivo de Bird fue mostrar que el criterio de "resolución de problemas", defendido por estos autores, es regresivo y, por ello, anti-intuitivo. En este texto analizo los argumentos de Bird en contra de estos autores y muestro en qué fallan. Posteriormente, se evalúa su postura a partir de sus propios fundamentos epistemológicos. Al final, presento algunas conclusiones que pueden inferirse del presente estudio.

Palabras clave: progreso científico, acumulación de conocimiento, resolución de problemas, inducción pesimista, verdad. 


\section{Abstract}

Recently, Alexander Bird (2007) suggested that science progresses when it shows "accumulation of justified knowledge". To validate his position, Bird has contrasted his ideas against the concepts of scientific progress developed by Thomas S. Kuhn and Larry Laudan, respectively. Bird's aim is showing that "problem-solving" criteria defended by Kuhn and Laudan, is regressive and, therefore, anti-intuitive. In this text I analyze Bird's arguments and I show why his position is untenable. Afterwards, I evaluate Bird's position from his own epistemological foundations. At the end, I present some of the conclusions that can be inferred from this analysis.

Keywords: Scientific progress, Accumulation of knowledge, Problem solving, Pessimistic induction, Truth.

\section{Introducción}

G $\mathrm{n}$ un extenso artículo publicado en 2007 y en otros dos artíCulos subsecuentes (2008 y 2010), Alexander Bird desarrolló lo que denomina la postura "epistémica" del progreso científico. De acuerdo con esta postura, existe progreso científico cuando se acumula conocimiento suficientemente justificado mediante la utilización de una metodología confiable. La estrategia que utilizó Bird para defender sus ideas fue contrastar éstas con los conceptos sobre el progreso científico propuestos desde otras posturas, específicamente la que Bird llama "semántica", desarrollada por Ilkka Niiniluoto, y las que llama "funcionalistas-internalistas", desarrolladas por Thomas S. Kuhn y Larry Laudan, respectivamente. ${ }^{1}$

${ }^{1}$ En este texto analizaré el progreso científico desde el punto de vista cognitivo, por lo que de aquí en adelante cuando me refiera al "progreso científico" lo estaré haciendo en relación al progreso cognitivo que exhibe la ciencia y no a otros tipos de progreso científico, como pueden ser el progreso tecnológico o el progreso educativo de la ciencia. 
Recientemente, Ilkka Niiniluoto (2014) y Gustavo Cevolani y Luca Tambolo (2012) mostraron que los argumentos elaborados por Bird en contra de la postura semántica son insostenibles. En particular, sugirieron que la idea de "progreso científico" tiene dos formas de interpretarse desde el punto de vista del concepto de verosimilitud: como "progreso real", el cual está construido a partir del incremento de la verosimilitud de las teorías científicas, y como "progreso estimado", el cual está concebido a partir del incremento de su verosimilitud estimada. De acuerdo con estos autores, el concepto de verosimilitud, entendido a partir de estas dos vertientes, explica -en contra de lo que argumentó Bird- cómo las creencias científicas están basadas en evidencia confiable y por qué tales creencias tratan de excluir la falsedad y preservar la verdad.

Sin embargo, Cevolani y Tambolo consideraron que los argumentos que Bird construyó en contra de las posturas funcionalistas-internalistas son "sustancialmente correctos" (2012: 924). En este sentido, el objetivo central del presente texto es mostrar que los argumentos de Bird en contra de las posturas funcionalistasinternalistas también son insostenibles, aunque por razones por supuesto distintas. El principal argumento de Bird en contra de Kuhn y Laudan está dirigido al criterio de "resolución de problemas" que estos autores propusieron para evaluar el progreso científico. Según Bird, este criterio es regresivo y anti-intuitivo, lo que mostraría por qué la acumulación en el poder de resolución de problemas que exhibe la ciencia no es una condición suficiente para explicar el progreso científico (Bird, 2007). Como veremos en la cuarta sección, el argumento que desarrolló Bird para sostener esta idea es cuestionable.

La estrategia argumentativa de Bird fue evaluar el criterio funcionalista-internalista de "resolución de problemas" a partir de algunos criterios epistemológicos que él favorece. Como es sabido, Kuhn y Laudan rechazaron que la ciencia exhiba "acumulación 
de conocimiento" cuando éste es entendido de manera tradicional como la "obtención de la verdad". Bird aseguró que este rechazo está erróneamente fundamentado en dos argumentos principales: (1) el argumento de la inducción pesimista y (2) la trascendencia del concepto de verdad. Son varios los contra-argumentos que Bird construyó para afirmar que la formulación de (1) tal y como la entendieron Kuhn y Laudan es débil y que (2) no requiere de una justificación correspondentista de la verdad, como sostuvo Kuhn, sino de una justificación a la mejor explicación. En la segunda sección de este texto, analizaré cada uno de estos argumentos. Como se verá, algunos de éstos requieren de análisis más detallados, mientras que otros, por su propia naturaleza, pueden ser refutados más rápidamente.

Por otro lado, Bird también afirmó que ambas posturas, la epistémica que defiende y la funcionalista-internalista, coincidirían si: (3) todo el conocimiento científico fuera reducible a conocer la solución de algunos problemas científicos y (4) si resolver un problema científico fuera un asunto de adquisición de un cierto tipo de conocimiento. Ambos puntos son rechazados por Bird (2007). En las secciones tercera y cuarta trataré de mostrar que también estos argumentos de Bird en contra de Kuhn y Laudan pueden ser rechazados por las posturas funcionalistas-internalistas. En la quinta sección dejaré de lado los argumentos de Bird en contra de Kuhn y Laudan para analizar su postura sobre el progreso científico en sí misma. En esta sección trataré de mostrar que sus ideas exhiben algunas falacias argumentativas que debilitan su postura. Finalmente, en la sexta sección ofreceré algunas conclusiones que se derivan del presente estudio. 


\section{El argumento de la inducción pesimista}

\section{y el de la trascendencia del concepto de verdad de Bird}

Thomas S. Kuhn y Larry Laudan coincidieron en que la meta cognitiva más importante de la ciencia es la resolución de problemas científicos (Kuhn, 1970; Laudan, 1977). ${ }^{2}$ En sus primeros trabajos, Kuhn propuso el concepto de "resolución de problemas" como criterio del progreso científico en el marco de su teoría sobre las revoluciones científicas y el cambio paradigmático, mientras que Laudan lo hizo desde la defensa de un instrumentalismo metodológico. ${ }^{3} \mathrm{Al}$ igual que Kuhn y Laudan, Bird también desarrolló una postura teleológica del progreso científico, pero sostuvo, a diferencia de estos autores, que la meta epistémica de la ciencia es la "producción de conocimiento". Cito a Bird al respecto: "La ciencia (o un campo o teoría científica particular) progresa precisamente cuando muestra acumulación de conocimiento científico" (2007: 64).

A pesar de que Bird aceptó que el concepto de conocimiento no puede ser definido de manera no circular, también afirmó que di-

${ }^{2}$ Cabe mencionar que el concepto de resolución de problemas no es una idea original de Kuhn ni de Laudan. Esta idea puede remontarse a los trabajos de C. S. Peirce y W. Whewell, como lo ha hecho ver Niiniluoto (1984). Posteriormente, esta idea fue retomada por Karl. R. Popper, entre otros autores.

${ }^{3}$ Quiero señalar aquí que para hacer un análisis integral de las ideas de ambos autores, tenemos que tomar en cuenta las diferentes etapas metodológicas por medio de las cuales estos autores desarrollaron sus ideas. Ciertamente no es lo mismo analizar a Kuhn desde la perspectiva de la Estructura de las Revoluciones Científicas que escribió en 1962, que veinte años después; por ejemplo, desde su texto "Commensurability, Comparability, Communicability" de 1982. Lo mismo sucede con Laudan quien claramente experimentó un cambio que va de la metodología historiográfica, que desarrolló en 1977, al naturalismo normativo, que propuso en 1987. Sin embargo, Bird no hace esta distinción metodológica en sus críticas a estos autores, por lo que en este texto sólo se hará referencia a estas diferentes etapas metodológicas cuando sea necesario para nuestra argumentación. 
cho concepto tiene un papel explicativo central para la mejor comprensión del progreso científico. A este respecto, Bird construyó tanto la gráfica que ilustra la concepción "ordinaria" o "intuitiva" que según él tenemos del progreso científico como la gráfica que ilustra el cambio en el conocimiento que puede experimentar la ciencia. Cuando se comparan ambas gráficas, éstas coinciden y lo hacen porque nuestra concepción "intuitiva" del progreso científico es, sostuvo Bird, "coextensional al concepto de crecimiento del conocimiento científico” (2007: 72).

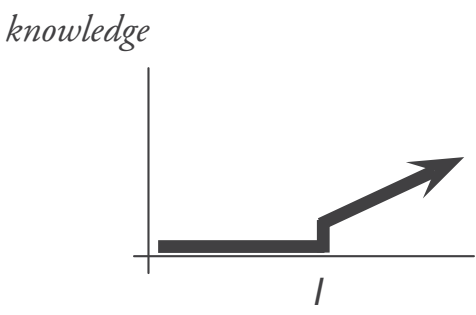

(b) progress

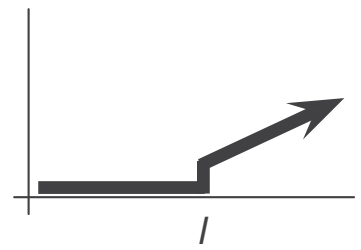

(c)

Figura 1. Gráfica de la concepción intuitiva del progreso cientifico $y$ del cambio del conocimiento (Bird, 2007: 66) ${ }^{4}$

Según la gráfica anterior, el progreso científico es co-extensional al crecimiento del conocimiento científico. Ahora bien, Bird sostuvo que la ciencia crece localmente cuando "alguna proposición científica llega a ser conocida” (2007: 76). Por lo tanto, el progreso científico se obtiene cuando una proposición científica llega a ser conocida. Sin embargo, al tomar en cuenta los contra ejemplos construidos por Gettier, el mismo Bird aceptó que el concepto

${ }^{4}$ Dado que el término progreso proviene del latín progressus que significa "avance", esto es, la acción de ir hacia adelante; el concepto mismo de progreso implica una transición desde un punto de referencia (b), en el caso del conocimiento, y (c), en el caso del progreso, hacia otro punto distinto de (b) y (c). En la gráfica, la transición está representada por la flecha ascendente. 
de conocimiento no puede definirse simplemente como "creencia verdadera justificada", dado que se puede tener creencias que sean accidentalmente verdaderas y estén justificadas. Tales casos "accidentales" no contribuirían al progreso científico, sostuvo Bird. Por lo tanto, las únicas proposiciones científicas que pueden ser conocidas -y de este modo contribuir al progreso científico- son las que están "suficientemente bien fundamentadas por la evidencia" y que han sido "apropiadamente confirmadas" (Bird, 2007: 77-79).

Para defender su postura, Bird contrastó sus ideas con los conceptos sobre el progreso científico construidos por Kuhn y Laudan, respectivamente. El objetivo de Bird fue mostrar, por un lado, que "nuestras intuiciones sobre el progreso en episodios posibles de cambio implican que las caracteristicas epistémicas son esenciales al progreso" (2007: 65; las cursivas son mías); por otro lado, que el criterio de resolución de problemas, propuesto por Kuhn y Laudan desde las posturas funcionalistas-internalistas ${ }^{5}$ que defendieron, es anti-intuitivo. Según Bird, la intuición nos indica que el progreso científico no puede ser regresivo, lo que asegura se sigue de las posturas defendidas por Kuhn y Laudan. Esta regresión, a la que apela Bird, mostraría por qué la acumulación en el poder de resolución de problemas no es una condición suficiente para explicar el progreso científico.

${ }^{5}$ Bird considera que las posturas teleológicas de Kuhn y Laudan son "funcionalistas" porque sus conceptos de progreso tienen que ver con el éxito que exhibe la ciencia en realizar una función, esto es: resolver problemas; por otro lado, son "internalistas" porque el juicio en relación a si la ciencia ha realizado o no progreso no depende de factores externos a la comunidad científica del campo en cuestión (Bird, 2007). Como sugirió un dictaminador (a) anónimo, conviene señalar que ciertamente concebir la "resolución de problemas" como una "función" de la ciencia es distinto a concebirla como una "meta" dado que el primer concepto enfatiza un proceso, mientras que el segundo enfatiza un fin: el éxito en la realización de este proceso. Para los objetivos de este trabajo, basta con señalar aquí esta diferencia. 
Como se sabe, Kuhn y Laudan rechazaron la idea de que la ciencia pueda alcanzar -o aproximarse a- la "verdad" (Kuhn, 1970; Laudan, 1977). En términos epistémicos, rechazaron que la ciencia exhiba "acumulación de conocimiento" cuando éste es entendido de manera tradicional como la "obtención de la verdad". Bird aseguró que el rechazo de Kuhn y Laudan está erróneamente fundamentado en dos argumentos, a saber:

(1) El argumento de la inducción pesimista.

(2) La trascendencia del concepto de verdad.

A continuación examinaré estos dos argumentos con el objetivo de mostrar en qué fallan.

\section{1. El argumento de la inducción pesimista}

Bird formuló en los siguientes términos el argumento de la inducción pesimista: es "la inferencia que se hace a partir de la premisa de que todas las teorías pasadas han sido falsadas hacia la conclusión de que todas las actuales y futuras teorías también serán falsadas" (2007: 73). Bird aseguró que el argumento de la inducción pesimista es un argumento débil por cuatro razones principales, a saber:

(a) Existen "venerables" ejemplos de proposiciones científicas que nunca han sido falsadas.

(b) Las teorías científicas sucesoras durante el cambio teórico no se crean de manera independiente de los éxitos y fracasos que exhiben las teorías que se abandonan.

(c) De la sucesión de teorías falsas, $T_{1} \ldots T_{n}$, no podemos concluir sin información adicional que las teorías futuras también serán falsas.

${ }^{6}$ El origen del término inducción pesimista puede ser rastreado hasta los trabajos de Henri Poincaré quien habló acerca de la "bancarrota de la ciencia" y de la "apilación de ruinas sobre ruinas" (Poincaré, 1952). Sin embargo, el uso moderno del término está identificado con los trabajos de Hilary Putnam (1978) y Larry Laudan (1981). 
(d) Incluso una sucesión de teorías falsas puede permitir el conocimiento de manera negativa (Bird, 2007). Revisemos cada uno de estos argumentos.

\section{Argumento 2. 1. a}

En relación a este argumento, Bird ofreció algunos ejemplos de proposiciones científicas que "nunca han sido falsadas". Dos de los ejemplos más interesantes son: "El agua es un compuesto de hidrógeno y oxígeno" y "los electrones están cargados negativamente" (2007: 73).

Exploremos el primero de estos dos ejemplos. La proposición "El agua es un compuesto de hidrógeno y oxígeno" contiene un concepto central, el de compuesto, que requiere explicitarse. El concepto de compuesto no siempre tuvo una connotación clara. A este respecto, no fue sino hasta alrededor de 1787, cuando Antoine Lavoisier (junto con otros químicos de su época) propuso reformar la nomenclatura de su tiempo, que el concepto de compuesto reflejó sus "composiciones elementales" (vid. Findlay, 2006). En otras palabras, ser un "compuesto" significó poseer tales o cuales elementos composicionales. En el caso específico del agua, en 1808, John Dalton todavía creía que la composición elemental del agua era de un solo átomo de hidrógeno y uno de oxígeno, esto es: $\mathrm{HO}$ (vid. Chalmers, 2009). Ahora bien, si se transforma la proposición de Bird, "el agua es un compuesto de hidrógeno y oxígeno", a los términos daltonianos de 1808 a partir del 'contenido' del concepto compuesto anteriormente expuesto, entonces esta proposición podría leerse de la siguiente manera:

$\mathrm{El}$ agua es un compuesto cuyos componentes elementales son $\mathrm{HO}$.

Si transformamos la misma proposición de Bird en los términos actualmente aceptados, entonces dicha proposición tendría que leerse de la siguiente manera: 
El agua es un compuesto cuyos componentes elementales son $\mathrm{H}_{2} \mathrm{O}$.

Así entendido, claramente puede verse que la proposición que sugiere Bird posee un contenido epistémico diferente en los términos daltonianos de 1808 y en los términos actuales. En otras palabras, la proposición daltoniana ha sido falsada por el contenido cognitivo de la misma expresión en los términos actualmente aceptados.

Exploremos ahora el segundo ejemplo de Bird. Después de que en 1888 Heinrich Hertz produjo las primeras ondas de radio, se volvió normal atribuir su espectro de emisión a la radiación causada por la oscilación de partículas cargadas. Notemos que en este momento todavía no se establecía el tipo de carga a la que se hacía referencia. Más tarde, en 1897, Pieter Zeeman trabajó en las predicciones de $\mathrm{H}$. A. Lorentz al respecto de la polarización de la luz y obtuvo un valor e/m para la oscilación de estas partículas, creándose una fuerte evidencia experimental (apoyada por otros trabajos como los de Emil Wiechart y Walter Kaufmann) que justificaba la creencia de que las partículas cargadas que vibraban eran la fuente del espectro del sodio y del cadmio. Zeeman concluyó, a partir de la dirección de la polarización de la luz asociada a las líneas espectrales, que las vibraciones de las partículas estaban negativamente cargadas. Posteriormente, J. J. Thomson trabajó en los rayos catódicos y otros fenómenos asociados, que comenzaron a configurar lo que más tarde sería conocida como la "teoría del electrón” de Lorentz, estableciéndose que éstos tienen una masa y una carga medibles.

Sin embargo, en 1906 Thomson todavía no utilizaba el concepto de electrón para referirse a estas partículas sub-atómicas con carga negativa, refiriéndose a éstas como corpúsculos. En realidad, fue George Johnstone Stoney quien creó el concepto de electrón para referirse a la unidad de carga de estas partículas (que como 
he mencionado es negativa) y no fue sino hasta 1899 que Lorentz adoptó el término para referirse al tipo de partículas a las que anteriormente había llamado iones. En otras palabras, no fue sino después de los experimentos realizados por Thomson que el concepto electrón se identificó con las partículas sub-atómicas cargadas negativamente. Por lo anterior, la proposición "los electrones están cargados negativamente", a la que hace referencia Bird, es una especie de verdad analítica que simplemente no puede ser falsada ya que el concepto mismo de electrón significa ser una partícula negativamente cargada, según la perspectiva histórica anteriormente referida.

Como puede inferirse del análisis de estos dos ejemplos, me parece que ninguna de las dos proposiciones construidas por Bird invalida la tesis de la inducción pesimista. ${ }^{7}$

\section{Argumento 2. 1. $b$}

Bird afirmó que las teorías científicas sucesoras durante el cambio teórico no se crean de manera independiente de los éxitos y fracasos que exhiben las teorías que se abandonan. Ciertamente este es el caso y tanto Kuhn como Laudan lo aceptaron, ya que ambos autores consideraron que, durante el cambio paradigmático o de tradiciones de investigación cientifica, algunos problemas que se consideraron importantes así como algunas de las soluciones que se idearon para ciertos problemas se conservaron, mientras que otros se desecharon (vid. Kuhn, 1970; 1977; Laudan, 1977). No obstante, el punto clave de la argumentación la hizo Laudan en 1981 cuando sostuvo que el éxito empírico que puede exhibir una teoría científica no está necesariamente vinculado a la verdad o

${ }^{7}$ Bird ofreció más ejemplos de proposiciones que supuestamente "nunca han sido falsadas" y que pueden, no obstante, ser refutadas de manera similar (vid. 2007: 73). 
aproximación a la verdad de dicha teoría debido, entre otras cosas, a que han existido teorías que hoy sabemos que son falsas y que no obstante tuvieron un alto grado de éxito empírico en diferentes aplicaciones. Ejemplos de este tipo de teorías son la mecánica, de Newton, la óptica de ondas o las leyes de la termodinámica. Por lo anterior, que una teoría $\mathrm{T}_{2}$ herede o recoja parte del éxito de la teoría precedente $\mathrm{T}_{1}$, no invalida el argumento de la inducción pesimista, ya que tanto $T_{2}$ como $T_{1}$ pueden de hecho ser falsas. ${ }^{8}$

\section{Argumento 2. 1. c}

Bird sostuvo que de la sucesión de teorías falsas, $T_{1} \ldots T_{n}$, no puede concluirse sin información adicional que las teorías futuras también serán falsas. Me parece claro que se requerirá de información adicional para establecer en su momento que $\mathrm{T}_{\mathrm{n}+1}$ también es falsa. No obstante, el punto se encuentra en que lo único que afirma el argumento de la inducción pesimista es que dada la experiencia pasada podemos inferir ahora que las teorías futuras probablemente también terminarán siendo falsas. Se trata tan sólo de una probabilidad inductiva que no se ve afectada por el hecho futuro de que se requiera de información adicional para aceptar o rechazar una teoría. Si en el futuro los científicos no contaran con la información empírica -o de otro tipo-adicional pertinente para

${ }^{8}$ Por supuesto, muchos autores han tratado de refutar el argumento de la inducción pesimista al sostener, por ejemplo, que la estructura matemática de las teorías científicas sí se ha retenido a través de las revoluciones científicas (Worral, 1989), o bien, que son los enunciados de Ramsey los que se preservan en la transición teórica y que éstos son los portadores del contenido cognitivo de las teorías científicas (Papineau, 2010); otros han afirmado que algunas partes de las teorías son las responsables del éxito empírico de una teoría y que otras partes sí pueden desecharse (Kitcher, 1993; Psillos, 1999; Leplin, 1997), etc. Actualmente, existen diversas referencias en torno a este debate, que por razones de espacio no pueden ser analizadas en este texto (vid. Mizrahi, 2013; Wray, 2013; Park, 2011; Frost-Arnold, 2011; Guillaumin, 2010; entre otros). 
rechazar una teoría específica, llamémosle $T^{*}$, seguramente $T^{*}$ se conservará -como se conservó por mucho tiempo la cosmología aristotélica-ptolemaica, por ejemplo-; también, supongo que dependiendo del período de tiempo en que se siga considerando $\mathrm{T}^{*}$ como no falsada, el argumento de la inducción pesimista perderá credibilidad.

\section{Argumento 2. 1.d}

Por último, sobre la afirmación de Bird de que "una sucesión de teorías falsas puede permitir el conocimiento de manera negativa" (2007: 80), me parece que es una aserción correcta desde el punto de vista de la postura epistémica que defiende el investigador, esto es: saber que estamos equivocados seguramente es una forma de evitar el error en el futuro. De igual manera, en los términos funcionalistas-internalistas que estamos analizando, saber que $\mathrm{X}$ representa un tipo de problema científico no resuelto, seguramente le indica a los científicos una línea futura de investigación. ${ }^{9}$ Sin embargo, no veo en qué sentido esta afirmación pueda socavar la inducción pesimista. Recordemos que el argumento de la inducción pesimista afirma como premisa que todas las teorías pasadas han sido falsadas y, hasta donde sé, nadie que haya defendido el argumento de la inducción pesimista se ha pronunciado en contra de que enterarnos de nuestros errores puede ser una manera de tratar de evitarlos en el futuro.

\footnotetext{
${ }^{9}$ Sobre este respecto, me parece que Thomas Nickles tiene razón cuando señala que el reconocimiento y formulación adecuada de un problema científico es ya de por sí un logro cognitivo de no poca importancia (Nickles, 1980).
} 


\section{2. El argumento de la trascendencia del concepto de verdad}

En contra de la postura anti-realista de Kuhn y Laudan, Bird afirmó que una concepción correspondentista de la verdad tradicionalmente ha sido atacada utilizando argumentos de tipo escéptico. Por ejemplo, se ha argumentado que si la verdad del enunciado $S$ es un asunto de "coincidencia" o "empate" entre el enunciado $S$ $y$ el hecho $F$, entonces saber que $S$ es verdadero tiene que ver con que de alguna manera inmediata sabemos que es el caso que la coincidencia ocurre. Sin embargo, para saber esto -argumentan los escépticos- tendríamos que tener un acceso al hecho $\mathrm{F}$ de manera independiente del enunciado $S$, lo que niegan los escépticos. Para rechazar el argumento escéptico, Bird sostuvo que el conocimiento de la verdad de $S$ no requiere de un acceso inmediato al conocimiento de dicha coincidencia, sino que dicho acceso puede estar mediado por una inferencia a la mejor explicación (IBE, por sus siglas en inglés) en el sentido de que la "coincidencia" entre $S$ y $F$ puede ser defendida argumentando que es la mejor explicación de la verdad de las proposiciones que pueden ser deducidas de $S$ (Bird, 2007).

Considero que el argumento de Bird es poco convincente para sus detractores porque pretender explicar la "coincidencia" entre $S$ y $\mathrm{F}$ argumentando que es la mejor explicación posible de la verdad de $S$, o de las proposiciones que pueden deducirse de $S$, puede ser una explicación aceptable para los defensores de posturas realistas de la ciencia, pero no necesariamente para los defensores de posturas escépticas como Laudan, con quien discute Bird. No obstante, incluso si se le concediera a Bird la posibilidad de explicar la verdad de las proposiciones que pueden deducirse de $S$ a partir de la supuesta "coincidencia" entre $S$ y F, no explicaría cómo y por qué $S$ y $F$ de hecho coinciden, al menos que se acepte $a$ priori una defi- 
nición tarskiana de la verdad o algo similar. ${ }^{10}$ En las siguientes dos secciones analizaré los últimos dos argumentos que Bird construyó en contra de las posturas funcionalistas-internalistas defendidas por Kuhn y Laudan.

\section{Argumento de la reducción del conocimiento científico}

Bird sostuvo que no toda la actividad científica que puede llamarse progresiva puede reducirse a resolver problemas. Para defender su punto, Bird cita el ejemplo de algunos astrónomos y naturalistas de los siglos XVIII y XIX que se pasaron la vida recolectando datos, ya sea sobre estrellas y cometas o bien sobre nuevas especies, sin resolver nunca ningún problema (Bird, 2007: 68). Sobre este respecto, me parece que es poco probable que la recolección de datos científicos se realice completamente al margen de algún marco teórico-conceptual mínimo que indique a los científicos el tipo de datos empíricos que es pertinente y relevante para un campo científico particular. ${ }^{11}$ Más aún, muchas veces los datos científicos

${ }^{10}$ Por supuesto, el debate en torno a los argumentos IBE es un debate abierto que no puede abordarse en este texto. Para nuestros fines, basta con señalar algunas de las problemáticas que enfrenta el argumento de Bird tal y como lo ha expuesto.

${ }^{11}$ Estoy consciente que se han desarrollado diversos estudios que han tratado de mostrar la relativa independencia teórico-conceptual de ciertas prácticas científicas, como es el caso de Ian Hacking que, en su libro Representing and Intervening, afirmó que "mucha de la investigación verdaderamente fundamental precede a la formulación de alguna teoría relevante" (1983: 158; las cursivas son mías). Más recientemente, Sergio Martínez (1993a; 1993b), siguiendo esta línea argumentativa, sostuvo que "el progreso cognoscitivo consiste en la evolución de conceptos asociada con la construcción de una jerarquía de fenómenos en la ciencia" (1993b: 12); no obstante, Martínez acepta que "la creación de un fenómeno puede hacerse al margen de una teoría, pero siempre implica conceptos" (1993b: 10). En otras palabras, ambos autores parecen aceptar que las prácticas científicas no son completamente independientes de aspectos teóricos o con- 
recolectados sirven para tratar de responder una pregunta o problema más fundamental o general.

Veamos un ejemplo: en el siglo XVII Thomas Simmons Mackintosh formuló una hipótesis que afirmaba que era la electricidad, y no la gravedad, la fuerza más importante en el universo. A este respecto, Mackintosh sostenía que "todos los cuerpos celestes que se mueven a través del sistema solar son afectados por la fuerza eléctrica" (1835b: 228). Algunos de los cuerpos celestes que más le interesaron a Mackintosh fueron los cometas, los cuales estaban constituidos por "inmensos volúmenes de materia con forma de aire que eran descargados por el Sol a través de la intervención de la electricidad" (1835a: 11). En 1835, Mackintosh registró la reaparición del cometa Haley con el objetivo de defender la idea de que los cometas eventualmente podrían condensarse, formar planetas y regresar al Sol. Lo que me interesa enfatizar con este ejemplo es que Mackintosh recolectó datos empíricos del paso del cometa Haley con el objetivo fundamental de responder, entre otras cosas, qué tipo de cosas eran los cometas según su teoría eléctrica del universo. Este ejemplo nos muestra que la recolección de datos a la que alude Bird difícilmente puede llevarse a cabo con sentido completamente al margen de una teoría (relevante o no) o de algún concepto teórico que guíe la investigación científica.

\section{Argumento de que resolver un problema científico no es equivalente a la adquisición de conocimiento}

Por último, pasemos al que a mi parecer es el más interesante argumento que Bird construyó en contra de las posturas defendidas

ceptuales. Cito nuevamente a Martínez: "Quizás valga la pena recalcar aquí que la noción de autonomía a la que nos referimos arriba no implica la falta de presuposiciones teóricas o más abiertamente metafísicas (como una concepción corpuscular de la materia, por ejemplo)" (1993a: 52). 
por Kuhn y Laudan. Bird afirmó que si resolver problemas científicos no implica la obtención de la verdad como aseguraron estos autores, entonces el criterio de resolución de problemas tampoco implica la obtención de conocimiento, cuando éste es entendido de manera tradicional como la obtención de la verdad (2007: 68). Según Bird, el modelo de Laudan sigue el modelo nomológico-deductivo de la explicación científica desarrollado por Carl Hempel (1965), quien afirmó que un fenómeno problemático $\mathrm{P}$ es resuelto por una teoría científica $\mathrm{T}$ cuando puede deducirse $\mathrm{P}$ de T.

Analicemos el ejemplo que construyó Bird de este caso: el filósofo natural medieval Nicole d'Oresme pensó que de la sangre caliente de un cabrío podrían extraerse diamantes (Oresme, 1968). Bird afirmó que si Oresme hubiera sido capaz de construir una teoría $\mathrm{T}$ a partir de la cual fuese posible deducir la extracción de diamantes de la sangre caliente de un cabrío, entonces dicha teoría T habría ofrecido una solución al problema que se planteó Oresme y, así, habría contribuido al progreso científico, según el modelo laudaneano-hempeliano del progreso científico. Ahora imaginemos a un estudiante, continúa Bird, quien muestra, digamos en $\mathbf{t}_{1}$, que la solución construida para solucionar el problema de Oresme, es falsa. Bird sostuvo que según la postura funcionalistainternalista de Laudan antes de $\mathbf{t}_{\mathbf{1}}$ la ciencia tenía una solución a un problema y, por ello, habría progresado; mientras que, acorde con la postura epistémica defendida por Bird, la situación es más bien la contraria, esto es, sólo después de $\mathbf{t}_{\mathbf{1}}$, momento en que el estudiante mostró que la solución era falsa, la ciencia progresó, aunque modestamente.

$\mathrm{El}$ argumento anterior me parece que tergiversa y omite algunos puntos centrales de las ideas, defendidas particularmente por Laudan, por las siguientes tres razones: (i) las soluciones a los problemas científicos, según el modelo de Laudan, no son verdaderas ni falsas, cito a Laudan al respecto: 
Sostendré que una teoría puede resolver un problema aun cuando sólo lo haya establecido de manera aproximada; para establecer si una teoría resuelve un problema, es irrelevante si la teoría es verdadera o falsa o si está bien o pobremente confirmada (1977: 22-23).

Por lo tanto, el hipotético estudiante sólo pudo haber mostrado que la solución de Oresme era falsa en los términos epistémicos que Bird defiende, pero no en los términos funcionalistas-internalistas con los que discute Bird. Ahora bien, (ii) si Oresme hubiera sido capaz de construir una teoría con la cual fuese posible deducir la extracción de diamantes de la sangre caliente de un cabrío, Bird asegura que según el criterio de resolución de problemas, dicha teoría habría ofrecido una solución al problema que se planteó Oresme y, de esta manera, habría contribuido al progreso científico, aunque en realidad se tratara de "sumar falsedades a falsedades" (2007: 69), es decir, tanto el problema como la solución habrían sido falsos. No obstante, según Laudan, un problema científico es un problema como tal hasta que ha sido efectivamente resuelto y para que esto suceda el fenómeno o suceso científico debe estar suficientemente autentificado (Laudan, 1977). Por lo anterior, el problema que se planteó Oresme, en principio, no era un problema genuino al no poder haber sido resuelto de manera efectiva.

Como sugirió un dictaminador anónimo, conviene enfatizar que la parte esencial del argumento de Bird radica en su afirmación de que tanto Kuhn como Laudan suscribirían que el descubrimiento del hipotético estudiante marcaría un retroceso en el progreso científico. Según Bird, antes de $\mathbf{t}_{\mathbf{1}}$ la ciencia habría obtenido una solución, mientras que después de $\mathbf{t}_{\mathbf{1}}$ la ciencia habría perdido dicha solución, lo que muestra que la acumulación en el poder de resolución de problemas no es una condición suficiente para el progreso científico (Bird, 2007). Sin embargo, (iii) ni 
Kuhn ni Laudan negaron que durante el cambio paradigmático o de tradiciones algunos problemas que se consideraron importantes, así como algunas de las soluciones que se ofrecieron a ciertos problemas, pueden dejar de ser vistos como problemas genuinos o soluciones pertinentes. A este respecto, Kuhn sostuvo que si bien durante las revoluciones científicas hay ganancias cognitivas, también hay pérdidas cognitivas importantes. ${ }^{12}$ Una de estas pérdidas es precisamente la eliminación de ciertos problemas antiguos y su sustitución por otros que emergen como relevantes para una comunidad particular en un momento específico. Asimismo, Laudan consideró que lo que cuenta como una solución a un problema empírico en un momento dado no necesariamente deberá contar como una solución aceptable en otro momento (Laudan, 1977).

En otras palabras, lo que Bird no alcanza a ver es que para las posturas funcionalistas-internalistas, la pérdida o abandono de algunos problemas y soluciones científicas particulares no implica la pérdida de la habilidad concreta -o poder, según la terminología de Bird- de la ciencia para la resolución de problemas. Por lo anterior, me parece que Bird comete la falacia de la composición al trasladar el caso contingente de que la ciencia no alcance a resolver un problema específico con la situación más general de que la ciencia pierda la habilidad para resolver problemas. ${ }^{13}$

Hasta aquí el análisis de los diversos argumentos que construyó Bird en contra de las posturas funcionalistas-internalistas. En

${ }^{12}$ De hecho, este rasgo del cambio paradigmático constituye el conocido tema de las "pérdidas cognitivas kuhnianas" (vid. Kuhn, 1970; 1977).

${ }^{13}$ Como señaló un dictaminador anónimo, las posturas funcionalistas-internalistas del progreso científico ciertamente tendrían que profundizar en ciertas cuestiones como la distinción entre el progreso metodológico y el progreso cognitivo que exhiben diferentes disciplinas científicas o responder a la pregunta de cómo podríamos medir el poder de resolución de problemas defendido por Kuhn y Laudan. Por supuesto, resolver aquí estos y otros problemas nos alejaría de los objetivos de este texto. 
la siguiente y última sección, analizaré cuáles son algunos de los problemas que enfrenta por sí misma la postura epistémica del progreso científico de Bird.

\section{Algunos problemas de la postura del progreso científico de Alexander Bird}

El primer aspecto problemático de la propuesta de Bird es que su noción de progreso científico como crecimiento o aumento del conocimiento parece ser demasiado amplia al punto dar cabida a posiciones filosóficas contrarias, como lo admitió el propio Bird (2007). Para señalar dos ejemplos, su postura daría cabida a posiciones realistas sobre la ciencia que consideran que la historia de la ciencia, como un todo, es la historia de la acumulación de conocimiento genuino corroborable en la medida en que alguna disciplina científica haya contribuido con el conocimiento, aunque sea de manera mínima, en el área de su competencia; también daría cabida a posiciones anti-realistas sobre la ciencia, como la defendida, por mencionar un ejemplo, por Bas Van Fraassen, quien seguramente suscribiría la idea de que la ciencia muestra aumento en el conocimiento de proposiciones científicas empíricas observacionalmente corroboradas (Van Fraassen, 1980)

Por lo anterior, me parece que la supuesta capacidad del criterio epistémico del progreso científico propuesto por Bird para contener posiciones filosóficas, incluso contrarias, más que una virtud ecléctica, como sugiere su autor, exhibe una desafortunada amplitud. Recordemos que las posturas eclécticas pretenden adoptar una postura intermedia que procura conciliar doctrinas opuestas. En este sentido, tanto las posturas realistas como las anti-realistas defienden la idea de que la ciencia obtiene conocimiento, en lo que no están de acuerdo es en el tipo de entidades, fenómenos, procesos o estructuras que la ciencia puede conocer. Afirmar que 
podemos explicar el progreso de la ciencia desde ambas posturas opuestas, porque ambas aceptan que la ciencia "acumula conocimiento", es anular el debate realista/anti-realista sobre el progreso científico.

Un segundo aspecto problemático de la postura epistémica de Bird es que, irónicamente, ésta parece no estar suficientemente justificada. Según Bird, existe progreso científico cuando se acumula conocimiento y se acumula conocimiento cuando las proposiciones científicas con las que se expresa la ciencia son verdaderas. A su vez, las proposiciones científicas son verdaderas cuando sabemos, afirma Bird, que están suficientemente justificadas. Dicha justificación se obtiene por vía de una metodología científica que nos proporciona evidencia epistémica confiable, la cual se obtiene cuando las proposiciones científicas están apropiadamente confirmadas (Bird, 2007). Sin embargo, ante un contra ataque de corte escéptico cuyo principal argumento fuera que los métodos científicos más básicos no pueden tener una justificación objetiva no circular, Bird parece que no podría aclarar cómo es que las proposiciones científicas llegan a estar apropiadamente confirmadas para que la evidencia epistémica sea confiable. La única sugerencia que hace al respecto recurre a lo que parece ser una justificación circular ya que propone que es la misma evidencia epistémica la que confirma las proposiciones científicas. La figura 2 muestra de manera gráfica la circularidad argumentativa de Bird: 
Acumulación de conocimiento

Existe acumulación de conocimiento cuando las proposiciones científicas son verdaderas

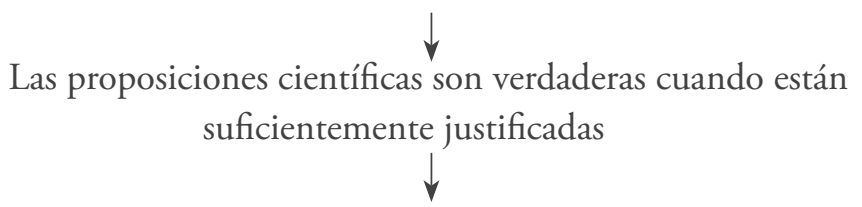

La justificación se logra vía una metodología científica que nos proporcione evidencia epistémica confiable

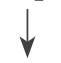

La evidencia epistémica confiable se logra cuando las proposiciones científicas están apropiadamente confirmadas

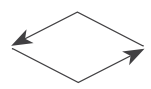

Las proposiciones científicas se confirman cuando existe evidencia epistémica confiable

Figura 2. Argumentación circular de Bird ${ }^{14}$

El tercer problema que señalaré aquí en relación a la propuesta de Bird es que ésta no logra establecer con claridad cómo se discierne el tipo de conocimiento que puede considerar progresivo del que

${ }^{14} \mathrm{El}$ ataque escéptico al confiabilismo de Bird aquí expuesto exige que todo argumento esté justificado, incluso los más fundamentales, lo que algunos podrían ver como un exceso. Por ejemplo, David Papineau apeló a una distinción entre "circularidad de regla" y "circularidad de premisa", concediendo que sólo ésta última es viciosa mientras que la primera puede ser aceptable (1992). Sin embargo, la circularidad de regla parece funcionar únicamente para aquellos que suponen que la inducción es confiable, esto es, sólo mediante la auto-proclamación. Por supuesto, abordar este problema excedería los límites de este trabajo, sin embargo, cabe señalar que el ataque escéptico es automáticamente atraído por Bird ante sus afirmaciones de corte confiabilista. 
no lo es. Intuitivamente, podemos considerar que no todo el conocimiento tiene el mismo valor en su contenido. Si esto es así, el conocimiento científico exhibe diferentes gradaciones que tendrían que sopesarse si Bird quiere evaluar objetivamente la progresividad científica. Desafortunadamente, Bird no dice nada sobre cómo puede ser evaluado el contenido del conocimiento científico.

Quiero seńalar, por último, el que me parece el problema intrínseco más importante de la propuesta sobre el progreso científico de Bird, a saber: su carácter irrefutable. Si uno se pregunta bajo qué condiciones el criterio epistémico de Bird no se cumpliría, la respuesta es que la única condición bajo la cual su criterio podría ser incorrecto es cuando lo que se creyó que era conocimiento en realidad no lo era, lo cual es imposible porque entonces, según afirma Bird, dicha creencia era, desde su mismo origen, falsa porque si progresar implica generar conocimiento y el conocimiento implica obtener verdades justificadas, entonces progresar implica la obtención de verdades justificadas. No obstante, si esto es así, todo episodio científico que visto retrospectivamente sea considerado como falso no será progresivo porque no habría constituido conocimiento genuino; mientras que, por el contrario, todo episodio que visto retrospectivamente sea considerado verdadero sería ipso facto progresivo. Por lo anterior, no es una casualidad que el criterio de progreso científico desarrollado por Bird siempre coincida con la gráfica de la figura 1 construida por él.

\section{Conclusiones}

Del anterior análisis podemos concluir que, cuando la ciencia resuelve un problema científico, se produce un tipo de conocimiento que no requiere del concepto de verdad, como afirman algunos defensores de posturas epistemológicas tradicionales del progreso científico como Alexander Bird. En otras palabras, las soluciones 
a los problemas científicos no son verdaderas ni falsas, sino soluciones adecuadas o inadecuadas para los fines cognitivos específicos que se persiguen. Por lo anterior, durante el cambio teórico es posible que algunos problemas que se consideraron importantes, así como algunas de las soluciones que se ofrecieron a ciertos problemas, pueden dejar de ser vistos como problemas genuinos o soluciones pertinentes; sin embargo, la pérdida o abandono de algunos problemas y soluciones científicas particulares no implica la pérdida de la habilidad concreta de la ciencia para la resolución de problemas. Por supuesto, es difícil calcular el número de problemas y soluciones que deben abandonarse para considerar que la habilidad en la resolución de problemas científicos se ha visto afectada. Asimismo, también es difícil calcular el peso cognitivo no sólo de los problemas científicos que enfrenta la ciencia, sino de la soluciones que se ofrecen para tales problemas. Sin embargo, lo importante aquí es puntualizar que dicho cálculo no puede hacerse con los criterios epistémicos tradicionales defendidos por Bird, como he tratado de mostrar a lo largo de este texto.

\section{Bibliografía}

Bird, Alexander, 2010, “The Epistemology of Science - A bird'seye View”, Synthese, núm. 175, pp. 5-16.

, 2008, "Scientific Progress as Accumulation of Knowledge: A Reply to Rowbottom", Studies in History and Philosophy of Science, 39, pp. 279-281. 89. , 2007, "What Is Scientific Progress?”, Nous, 41(1), pp. 64-

Cevolani, Gustavo y Tambolo, Luca, 2012, "Progress as Approximation to the Truth: A Defence of the Verisimilitudinarian Approach", Erkenntnis, 78, pp. 921-935. 
Chalmers, Alan, 2009, The Scientist's Atom and The Philosophers's Stone, Dordrecht, Springer.

Dalton, John, 1964, A new system of chemical philosophy, New York, Citadel Press.

Findlay, Robin, 2006, "Elements, Compounds, and Other Chemical Kinds", Philosophy of Science, 73 (5), pp. 864-875.

Frost-Arnold, Greg, 2011, "From the Pessimistic Induction to Semantic Antirealism", The Philosophy of Science, 78, pp. 11311142.

Guillaumin, Godfrey, 2010, "Progreso científico mediante convergencia cognitiva. Socavando la inferencia pesimista”, Revista Valenciana de Filosofía y Letras, 3 (5), pp. 89-116.

Hacking, Ian, 1983, Representing and Intervening, Cambridge, Cambridge University Press.

Hempel, Carl, 1965, Aspects of Scientific Explanation And other Essays in the Philosophy of Science, New York, The Free Press.

Kitcher, Philip, 1993, The Advancement of Science, Science without Legend, Objectivity without Illusions, Oxford, Oxford University Press.

Kuhn, Thomas S., 1982, "Commensurability, Comparability, Communicability”, en PSA: Proceedings of the Biennial Meeting of the Philosophy of Science Association, 2 vol., pp. 669-688.

, 1970, The Structure of Scientific Revolutions, Chicago, University of Chicago Press.

, 1977, The Essential Tension: Selected Studies in Scientific Tradition and Change, Chicago, University of Chicago Press.

Laudan, Larry, 1987, "Progress or Rationality? The Prospects for Normative Naturalism”, American Philosophical Quarterly, 24 (1), pp. 19-31. 
, 1981, "A Confutation of Convergent Realism”, Philosophy of Science, 48 (1), pp. 19-49.

, 1978, "The Philosophy of Progress", PSA: Proceedings of the Biennial Meeting of the Philosophy of Science Association, (2), pp. 530-547.

, 1977, Progress and Its Problems, Towards a Theory of Scientific Growth, Los Angeles, University of California Press.

Leplin, Jarret, 1997, A Novel Defense of Scientific Realism, Oxford, Oxford University Press.

Mackintosh, Simmons, 1835a, "Electrical Theory of the Universe", Mechanic's Magazine 24.635, (6 de octubre), p. 11.

,1835b, "Electrical Theory of the Universe", Mechanic's Magazine, 24.645, (19 de diciembre), pp. 227-234.

Martínez, Sergio, 1993a, "Método, Evolución y Progreso en la Ciencia", Critica, Revista Hispanoamericana de Filosofía, XXV (73), pp. 37-69.

, 1993b, "Método, Evolución y Progreso en la Ciencia", en Critica, Revista Hispanoamericana de Filosofía, XXV (74), pp. 3-21.

Mizrahi, Moti, 2013, "The pessimistic induction: a bad argument gone too far", Synthese, 190, pp. 3209-3226.

Nickles, Thomas, 1980, "Scientific Problems: Three Empiricist Models", PSA Proceedings of the Biennial Meeting of the Philosophy of Science Association, (1), pp. 3-19.

Niiniluoto, Ilkka, 2014, "Scientific Progress as Increasing Verisimilitude", Studies in History and Philosophy of Science, 46, pp. 73-77.

,1984, Is Science Progressive?, Dordrecht, Kluwer Academic Publishers. 
Oresme, Nicole, 1968, Nicole Oresme and the Medieval Geometry of Qualities and Motions. A Treatise on the Uniformity and Difformity of Intensities known as Tractatus de Configurationibus Qualitatum et Motuum, Marshall Clagett (ed.), Madison, University of Wisconsin Press.

Papineau, David, 2010, "Realism, Ramsey sentences and the pessimistic meta-induction", Studies in History and Philosophy of Science, 41, pp. 375-385.

Park, Seungbae, 2011, "A Confutation of the Pessimistic Induction", Journal for General Philosphy of Science, 42, pp. 75-84.

Psillos, Stathis, 1999, Scientific Realism: How Science Tracks Truth, Routledge, Taylor and Francis Group.

Poincaré, Henri, 1952, Science and hypothesis, New York, Dover Publications.

Putnam, Hilary, 1978, Meaning and the Moral Sciences, London, Routledge \& Kegan Paul.

Van Fraassen, Bas, 1980, The Scientific Image, Oxford, Oxford University Press.

Worrall, John, 1989, "Structural realism: The best of both worlds", Dialectica, 43 (1-2), pp. 99-124.

Wray, Brad, 2013, "The pessimistic induction and the exponential growth of science reassessed", Synthese, 190, pp. 4321-4330.

Recibido: 17 de septiembre de 2014; aceptado: 23 de noviembre de 2014 